\title{
Bioequivalence Evaluation of Two Formulations of Lamotrigine Tablets in Healthy Volunteers
}

\author{
Adriana Ruiz ${ }^{1 *}$, Fanny Cuesta ${ }^{2}$, Sergio Parra ${ }^{2}$, Blanca Montoya ${ }^{2}$, Margarita Restrepo ${ }^{1}$ Rosendo Archbold ${ }^{1}$, Lina Peña ${ }^{2}$ and Gloria Holguín ${ }^{1}$
}

${ }^{1}$ Department of Pharmacy, School of Pharmaceutical Chemistry, Universidad de Antioquia, Medellín, Colombia

${ }^{2}$ Department of Pharmacology, School of Medicine, Universidad de Antioquia, Medellín, Colombia

\begin{abstract}
Lamotrigine is a phenyltriazine used in the treatment of epilepsy and bipolar disorder type I. The purpose of this study was to compare the bioavailability in healthy Colombian volunteers of two brands of lamotrigine $100 \mathrm{mg}$ tablets: a new generic formulation (test product) developed by Humax Pharmaceuticals S.A (Medellín, Col) and LAMICTAL ${ }^{\circledR}$ (reference product) from Glaxo Operations UK Ltd (Ware, UK). A single-dose, randomized, two-period, two-sequence crossover study, with six weeks washout period, was performed. Blood samples were obtained from 0 to 144 hours after dosing and plasma lamotrigine levels were determined by a validated high performance liquid chromatographic (HPLC) method. The $90 \%$ confidence intervals (Cls) for the ratios of the In $\mathrm{AUC}_{0-\infty}$ and In $\mathrm{C}_{\max }$ means between the reference and test formulations were constructed under 80/125 rule for bioequivalence limit. Fourteen subjects were enrolled in the study, but only twelve completed both treatment periods. The estimated pharmacokinetic parameters of lamotrigine for the reference and test formulations were $C \quad 2.314 \pm 0.414 \mu \mathrm{g} / \mathrm{mL}$, $2.226 \pm 0.355 \mu \mathrm{g} / \mathrm{mL} ; A U_{0-120} 70.148 \pm 10.824 \mu \mathrm{g} . \mathrm{h} / \mathrm{mL}, 69.277 \pm 13.432 \mu \mathrm{g} . \mathrm{h} / \mathrm{mL}$, and for $\mathrm{AUC}_{0-\infty}$ were $78.524 \pm$ $16.000 \mu \mathrm{g} . \mathrm{h} / \mathrm{mL}, 77.532 \pm 15.255 \mu \mathrm{g} . \mathrm{h} / \mathrm{mL}$, respectively. The $90 \%$ Cls for the In-transformed ratio (test $/$ reference) of $\mathrm{AUC}_{0-\infty}$ and $\mathrm{C}_{\text {max }}$ were 88.97 to 110.65 and 87.77 to 106.37 , respectively.
\end{abstract}

Conclusions: In this single dose study it was found that the test and reference products of lamotrigine $100 \mathrm{mg}$ tablets complied with the regulatory criteria for equivalence with respect to rate and extent of absorption according to the guidances of Instituto Nacional de Vigilancia de Medicamentos y Alimentos (INVIMA) and FDA.

Keywords: Lamotrigine; Lamictal ${ }^{\circledR}$; Bioavailability; Bioequivalence; Pharmacokinetics; HPLC

\section{Introduction}

Epilepsy is one of the most common neurological disorders. It is usually controlled, but cannot be cured with medication. Although there are many anticonvulsant medications able to inhibit the development or spread of abnormal spontaneous electrical activity throughout the nervous system, several drugs are characterized by low effectiveness in certain epilepsy syndromes and a relatively high frequency of serious side effects. Newer anticonvulsants offer alternatives to replace or combine with older medications, therefore improving patient safety or enabling greater seizure control.

Lamotrigine was approved for the treatment of epilepsy by Food and Drug Administration (FDA) in 1994. It is currently used and recommended as monotherapy for partial seizures and bipolar disorder type I [1,2]. It may also be an option in add-on therapy for the treatment of partial seizures, generalized seizures of Lennox-Gastaut syndrome [3], and tonic-clonic seizure with primary generalization [4]. It has been proposed that the anticonvulsivant mechanism of action of lamotrigine consists in blocking voltage-gated sodium channels [5], thus inhibiting the release of the excitatory neurotransmitter glutamate [6].

Lamotrigine pharmacokinetics is characterized by complete absorption after oral administration, with an absolute bioavailability of $98 \%[7,8]$, reaching peak plasma concentration $\left(\mathrm{C}_{\max }\right)$ between 0.5 to 4.0 hours. The apparent volume of distribution of lamotrigine ranges from $1.0-1.4 \mathrm{~L} / \mathrm{kg}$ [9], its plasma protein binding is approximately $55 \%$ [9]. This drug is metabolized by glucuronic acid conjugation into inactive metabolites [10], and $94 \%$ of it is recovered in urine [8]. Elimination half-life of lamotrigine varies between 22.8 and 37.4 hours
[11] and the proposed therapeutic range for seizure control is 1 to 4 $\mathrm{mg} / \mathrm{L}[11]$ although this has not been well-established [12].

In Colombia, the Instituto Nacional de Vigilancia de Medicamentos y Alimentos (INVIMA) requires that manufacturers of anticonvulsant generic drugs ensure the quality of these lower cost alternatives through bioequivalence studies, before approving its marketing or renewal license. This strategy is based on the assumption that drugs that have similar plasma concentration profiles over time should produce a similar therapeutic effect.

This study was designed to assess the bioavailability and bioequivalence between a generic formulation of lamotrigine (test product) and the reference product LAMICTAL ${ }^{\circledR}$, after oral administration of a single-dose.

\section{Subjects and Methods}

\section{Study products}

Test product, LAMOTRIGINE $100 \mathrm{mg}$ immediate release tablets, was a mixture of the lots LP1607, LP1707 and LP1807 made on an equal number of tablets of each batch. The product was manufactured

*Corresponding author: Adriana Ruiz, Professor, Department of Pharmacy, Universidad de Antioquia, A.A.1226 Medellín, Colombia, Tel: 57 4-219 54 78; Fax 57 4-219 2195459; E-mail: aruiz@farmacia.udea.edu.co, adriana75m@yahoo.com

Received February 08, 2012; Accepted March 23, 2012; Published March 25, 2012

Citation: Ruiz A, Cuesta F, Parra S, Montoya B, Restrepo M, Archbold R, et al. (2012) Bioequivalence Evaluation of Two Formulations of Lamotrigine Tablets in Healthy Volunteers. J Bioequiv Availab 4: 030-034. doi:10.4172/jbb.1000107

Copyright: (c) 2012 Ruiz A, et al. This is an open-access article distributed under the terms of the Creative Commons Attribution License, which permits unrestricted use, distribution, and reproduction in any medium, provided the original author and source are credited. 
and provided by Humax Pharmaceutical S.A. (Medellín, Colombia). Reference product, LAMICTAL ${ }^{\circledR}$ lamotrigine $100 \mathrm{mg}$ dispersible tablets, lot R306554B, manufactured by Glaxo Operations UK Ltd. (Ware, UK) was purchased from a local pharmacy.

Before the bioequivalence study, the products were tested for potency, dissolution and dosage uniformity through analytical methods used by the manufacturer of the test product in the Laboratorio Especializado de Análisis of the Facultad de Química Farmacéutica, Universidad de Antioquia. The products were judged suitable for the bioequivalence study if the assayed potency of the test product not differ from that of the reference product by more than $5 \%$; the mean percentage dissolved in 30 minutes was not less than $80 \%$ and the acceptance value for dosage uniformity of the 10 dosage units is less than or equal to $15 \%$.

\section{Subjects}

Healthy Colombian volunteers, nonsmoking, aged 18 to 30 years, and within $15 \%$ of ideal body weight for height (Metropolitan Life Insurance Company Statistical Bulletin, 1983) [13] were eligible to be enrolled in the study. Inclusion criteria encompassed no evidence of cardiac, pulmonary, gastrointestinal, hepatic, renal, hematologic, or neurologic disorders, or any acute or chronic disease, no history of drug or alcohol addiction, normal laboratory tests (complete blood counts, urinalysis, liver and kidney function, and fasting blood sugar); and serological negativity HIV, hepatitis B and blood pregnancy test (for females).

Subjects were informed by an investigator about the purposes and risks of the study and written informed consent was obtained from all volunteers. They were asked to abstain from using concomitant medications, including over-the-counter products, dietary supplements and natural products, two weeks prior to dosing and throughout the end of the study. Caffeine and/or xanthine-containing products or alcohol were not allowed 2 days before the first administration of the study medications and throughout the blood sampling periods. Subjects were required to fast for at least 12 hours before each scheduled dosing.

\section{Study design}

The protocol and informed consent form were reviewed and approved by the Ethics Committee of the Facultad de Medicina, Universidad de Antioquia, Medellín, Colombia. The study was performed in compliance with the principles of the Declaration of Helsinki [14], the guidelines for Good Clinical Practice [15,16] and the Resolution No. 8430 of Ministerio de la Protección Social, República de Colombia [17].

This study was conducted as a single-dose, two treatment, twoperiod, two-sequence crossover design with a six weeks washout period. Using a table of random numbers, volunteers were assigned to receive lamotrigine $100 \mathrm{mg}$ test or reference tablet and personnel conducting the study and the analyses were blinded in treatment assignment.

\section{Study drug administration and blood sampling}

Subjects were confined to the hospital one-hour prior to dosing of lamotrigine throughout completion of the 24 hour blood sampling period. During the first hour a urine sample was taken from each volunteer to assess illicit drugs or alcohol intake, blood pressure and pulse rate were monitored, and an indwelling intravenous cannula was inserted into an antecubital vein. A dose of the test or reference products was administered with $240 \mathrm{~mL}$ of water at room temperature.

Volunteers remained in semi-sitting position three hours after drug administration to ensure adequate gastric emptying and were kept at rest or walking on small areas during the time of confinement. The blood pressure and pulse rate of each subject were monitored at 2, 8 and 24 hours after dosing. During hospitalization, volunteers were under continuous medical surveillance, and for the duration of the additional sampling and washout period subjects maintained contact with the investigators to report any adverse events.

Subjects were allowed to have a fruit juice one hour after drug administration. They had standardized meals at 2.5, 5, 8, 11, and 24 hour after dosing (breakfast, lunch, break, dinner and next day breakfast).

Blood samples were obtained at $0,0.3,0.6,1,1.3,1.6,2,2.5,3.5$, $6,12,24$ hours. Subjects later on went to the Pharmacology and Toxicology laboratory of the Facultad de Medicina, Universidad de Antioquia for further sampling at 48, 96, 120, and 144 hours after drug administration. Blood was collected into $10 \mathrm{~mL}$ heparinized vacuum tubes (Vacutainer, Becton, Dickinson and Company, Franklin Lakes, New Jersey). Samples were centrifuged at $2000 \mathrm{rpm}$ for 15 minutes, and then the plasma was aliquoted in two containers. One of them was frozen at $-20^{\circ} \mathrm{C}$ until analysis. The other aliquot was frozen at $-70^{\circ} \mathrm{C}$, as retention sample for a period of five years.

\section{Tolerability}

Subjects were monitored for adverse events during both periods of the study and additional sampling visits. Subjects were asked to report any undesirable sign or symptom occurring after the start of the study. Adverse events (AE) were collected based on interview and spontaneous reports and recorded on a case-report form. The study physicians graded the AEs as mild, (it interferes with daily activities), moderate (it interferes with daily activity but it is still able to do it), or severe (it is disabling and requires medical attention).

\section{Determination of plasma concentrations of lamotrigine}

Plasma concentrations of lamotrigine were determined by a HPLC method previously reported by Matar et al. [18] with minor modifications included chloroform/ethyl ether $(80 / 20, \mathrm{v} / \mathrm{v})$ as the extraction solvent, carbamazepine as the internal standard and $10 \mathrm{mM}$ potassium phosphate $(\mathrm{pH} 7.5)$-methanol-acetonitrile $(60: 21: 19)$ at a flow rate of $0.8 \mathrm{~mL} / \mathrm{min}$ as the mobile phase.

A liquid chromatographic system (model 1100, Agilent Technologies, Palo Alto, California) with photodiode array detector set at $220 \mathrm{~nm}$ was used. Chromatographic separation was performed on a 250 x 4.0 mm, 5 mm, C18 column (LiChrospher ${ }^{\circledR}$ RP-Select B, Merck, Darmstadt, Germany). Samples were kept at room temperature in the autosampler, and $100 \mu \mathrm{L}$ of solution was injected for analysis. Data acquisition and analysis were performed by ChemStation ${ }^{\circledR}$ software (Agilent Technologies, Palo Alto, California).

The Method was validated using established international guidelines [19]. Quality control samples (low $0.25 \mu \mathrm{g} / \mathrm{mL}$ and high 1.00 $\mu \mathrm{g} / \mathrm{mL}$ ) were included in each analytical run to assess its performance.

\section{Pharmacokinetic and statistical analysis}

Pharmacokinetic data were calculated by the non-compartmental method. The maximum plasma concentration $\left(\mathrm{C}_{\max }\right)$ and the time to 
reach it $\left(\mathrm{T}_{\max }\right)$ were determined by inspecting each individual plasma level - time curves. The elimination rate constant $\left(\mathrm{k}_{\mathrm{e}}\right)$ was obtained by ln-linear regression of the terminal decay phase. The area under the plasma level-time curve $\left(\mathrm{AUC}_{0-120 \mathrm{~h}}\right)$ was obtained by the trapezoidal rule, and the $\mathrm{AUC}_{12 \mathrm{~h}-\infty}$ time was determined by dividing the $120 \mathrm{~h}$ plasma concentration by $\mathrm{k}_{\mathrm{e}}$, and adding this result to the $\mathrm{AUC}_{0-120 \mathrm{~h}}$.

Analysis of variance (ANOVA) for the cross design (Statistica 6.0, Statsoft Inc, 2001) was performed on ln-transformed data of $\mathrm{AUC}_{0-\infty}$ and $\mathrm{C}_{\max }$ in order to assess the effects of treatment, period, sequence of administration.

The data was also analyzed in relation to the assumptions of the two-formulation, two-period, two-sequence, crossover design to assess: carry over effect, intra-subject variability, intra e inter-subjects residuals normality, residual independence and outliers for $t-r, t / r$ and $\ln t / r$ variables untransformed. Differences were considered statistically significant when the $p$ value was equal to or less than 0.05 .

To conclude bioequivalence the two - one side method suggested by Schuirmann [20] was used, taking into account the standard error of the ANOVA for each parameter. The $90 \%$ confidence intervals (CIs) for the ratios of the $\ln \mathrm{AUC}_{0-\infty}$ and $\mathrm{C}_{\max }$ means between the reference and test products were constructed under $80 / 125$ rule for bioequivalence limit.

Bioequivalence was accepted if theses CIs were within $80 \%$ and $125 \%$ of the reference mean.

\section{Results}

\section{Study products}

Results of potency, dissolution and dosage uniformity both the reference product and the test product are summarized in Table 1 . The test product was different from the reference product by $1.7 \%$. Both products met the requirements for the tests.

\section{Subjects}

The study was conducted in fourteen (14) Colombian subjects, six (6) men and eight (8) women aged between 19 and 26 years, with an average of $21.9 \pm 1.9$ years. Their weights ranged from 50.0 to $68.2 \mathrm{~kg}$, with an average of $59.3 \pm 5.5$, and height ranged between 1.55 and 1.78 $\mathrm{m}$, with an average of $1.65 \pm 0.08$.

In the second period, two subjects voluntarily withdrew, one belonging to the test product / reference product sequence and the other one to the inverse sequence. Statistical calculations were performed using data obtained from 12 volunteers who completed the test.

\section{Tolerability}

In the first period, one volunteer presented dizziness, nausea, vertigo and weakness. During the second period, another volunteer showed erythematous macules, not itchy, with irregular border in the anterior neck and chest.

\section{Bioanalytical method validation}

The analysis of six blank samples did not show interference in the chromatograms. The calibration curve of spiked plasma samples showed good linearity in the range of $0.10-2.5 \mu \mathrm{g} / \mathrm{mL}$. Correlation coefficient calculated from curves constructed for interday $(n=6)$ and intraday $(\mathrm{n}=6)$ evaluations were 0.9994 and 0.9996 , respectively.
The limit of quantification of the assay was $0.10 \mu \mathrm{g} / \mathrm{mL}$. Intraday accuracy (expressed on the basis of percent recovery) ranged from 95.4\% to $100.3 \%$, whereas intraday precision (expressed as coefficients of variation) ranged from $5.7 \%$ to $13.7 \%$. Interday accuracy ranged from $96.5 \%$ to $107.8 \%$, whereas interday precision ranged from $8.2 \%$ to $14.3 \%$. The result obtained from the stability study showed that lamotrigine in plasma was stable kept at $-20^{\circ} \mathrm{C}$ during the analysis period (4 months).

\section{Pharmacokinetic and statistical analysis}

The mean concentration-time profiles from the volunteers after oral administration of the products are shown in Figure 1 and 2 . The mean (standard deviation) of the parameters $\mathrm{AUC}_{0-\infty}, \mathrm{C}_{\max }$ and $\mathrm{T}_{\max }$ and other associated pharmacokinetic parameters are described in Table 2.

\begin{tabular}{|l|l|l|l|}
\hline Product & Potency & Dissolution & Dosage Uniformity \\
\hline Test & 99.9 & $93.5 \pm 2.4$ & 7.4 \\
\hline Reference & 101.6 & $91.3 \pm 9.2$ & 6.7 \\
\hline
\end{tabular}

Table 1: Results of potency (mean percentage of active ingredient per tablet) dissolution (mean percentage of active ingredient dissolved) and dosage uniformity (acceptance value in percentage) tests to the test and reference products.

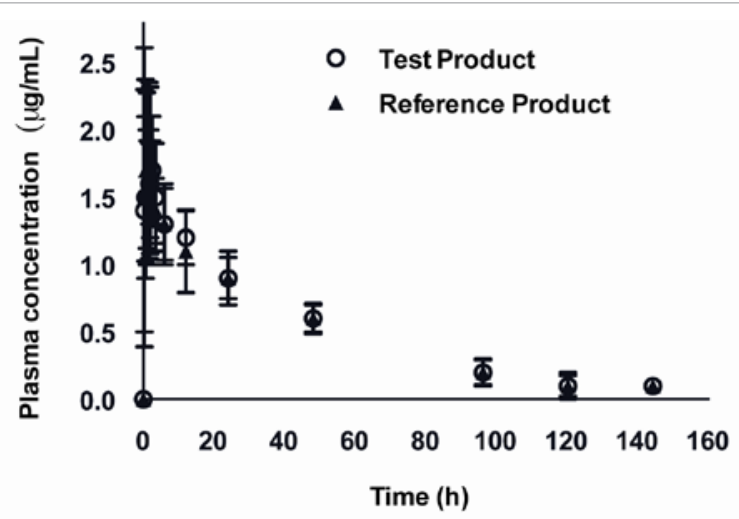

Figure 1: Mean $( \pm \mathrm{SE})$ plasma concentration-time curves from 0 to 144 hours after a single $100 \mathrm{mg}$ dose administration of a test and reference products of lamotrigine in 12 healthy Colombian volunteers.

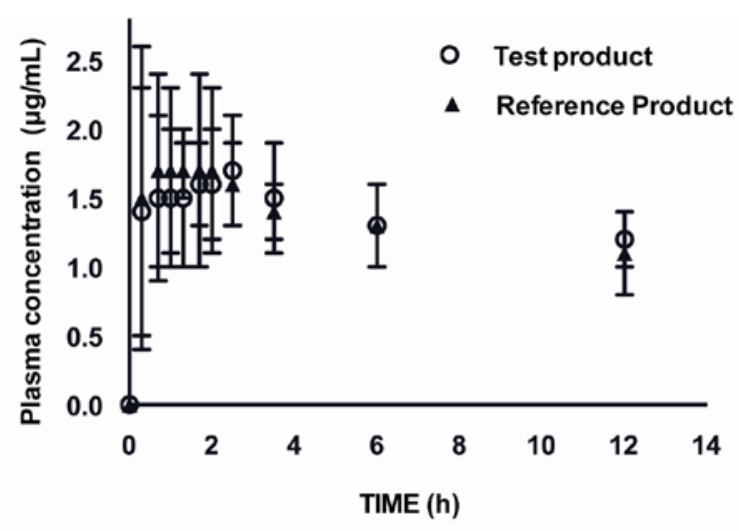

Figure 2: Mean $( \pm \mathrm{SE})$ plasma concentration-time curves from 0 to 12 hours after a single $100 \mathrm{mg}$ dose administration of a test and reference products of lamotrigine in 12 healthy Colombian volunteers. 
None of the effects examined with ANOVA for $\mathrm{AUC}_{0-\infty}$ and $\mathrm{C}_{\max }$ (treatment, period, sequence) were statistically significant. Similarly, carryover effect was no found. Intrasubject variation was similar in both formulations, regarding these two parameters. The intra and inter subject residuals can be assumed normal and independent. No outliers were detected.

The ratios between the test and reference products of $\ln \mathrm{AUC}_{0}$ and $\ln \mathrm{C}_{\max }$ means and the $90 \%$ CIs obtained are shown in Table 3.

\section{Discussion}

The main objective of bioequivalence studies is to assure the efficacy and safety of generic formulations. Therefore, two formulations of the same drug are considered to be bioequivalent and ergo therapeutically equivalent if they exhibit a comparable extent and rate of absorption, when they are administered in the same molar dose and under similar experimental conditions [21].

In this study, the test and reference products were evaluated to assess its "similarity" in vitro prior the bioequivalence evaluation to ensure their quality. The results of the tests showed that both products met the requirements (Table 1) and the differences found in the potency and dissolution test were small, indicating that probably the products would have a similar performance in vivo.

This study was performed as a pilot study with 12 subjects, to establish values for $\mu_{\mathrm{T}} / \mu_{\mathrm{R}}$ and $\sigma^{2}$ required to calculate the simple size according to the formula proposed by Julious [22], but once completed, and the parameters calculated, the sample size obtained was a total of six volunteers. In addition the posteriori calculated power of the study gave a value of $94 \%$. These results indicated that a sample size of 12 subjects is adequate to conclude bioequivalence between these two formulations.

No adverse events necessitating subject withdrawal from the study

\begin{tabular}{|l|l|l|}
\hline Parameter & Test product & Reference Product \\
\hline $\mathrm{C}_{\max }(\mu \mathrm{g} / \mathrm{mL})$ & $2.226(0.355)$ & $2.314(0.414)$ \\
\hline $\mathrm{T}_{\max }(\mathrm{h})$ & $1.380(1.060)$ & $0.933(0.729)$ \\
\hline $\mathrm{k}_{\mathrm{e}}(\mathrm{h})$ & $0.020(0.004)$ & $0.021(0.005)$ \\
\hline $\mathrm{t}_{1 / 2}(\mathrm{~h})$ & $36.261(6.801)$ & $35.694(9.476)$ \\
\hline $\mathrm{AUC}_{0-120}(\mu \mathrm{g} / \mathrm{mL}) \times \mathrm{h}$ & $69.277(13.432)$ & $70.148(10.824)$ \\
\hline $\mathrm{AUC}_{0-\infty}(\mu \mathrm{g} / \mathrm{mL}) \times \mathrm{h}$ & $77.532(15.255)$ & $78.524(16.000)$ \\
\hline
\end{tabular}

Table 2: Pharmacokinetic parameters (mean (SD)) of lamotrigine after a single $100 \mathrm{mg}$ oral dose of the two products in healthy Colombian volunteers $(n=12)$.

\begin{tabular}{|l|l|l|}
\hline Parameter & Ratio Test/Reference & IC 90\% \\
\hline $\mathrm{C}_{\max }(\mu \mathrm{g} / \mathrm{mL})$ & 0.9584 & $87.77 \%-106.37 \%$ \\
\hline AUC $_{0-\infty}(\mu \mathrm{g} / \mathrm{mL}) \times \mathrm{h}$ & 0.9982 & $88.97 \%-110.65 \%$ \\
\hline
\end{tabular}

Table 3: Average ratio of test product / reference product and confidence intervals $90 \%$ of In-transformed data for the parameters $C_{\max }$ and $A \cup C_{0-\infty}$.

\begin{tabular}{|l|l|l|l|l|}
\hline Pharmacokinetic parameters & \multicolumn{3}{|l|}{ Colombian population } & \multicolumn{2}{l|}{ Thai population } \\
\hline & Test & Reference & Test & Reference \\
\hline AUC $_{0-\infty}(\mu \mathrm{g} / \mathrm{mL} \cdot \mathrm{h}-1)$ & 77.5 & 78.5 & 74.9 & 74.3 \\
\hline $\mathrm{C}_{\max }(\mu \mathrm{g} / \mathrm{mL})$ & 2.2 & 2.3 & 1.7 & 1.7 \\
\hline $\mathrm{T}_{\max }(\mathrm{h})$ & 1.4 & 0.9 & 1.2 & 1.4 \\
\hline $\mathrm{T}_{1 / 2}(\mathrm{~h})$ & 36.3 & 35.7 & 35.0 & 34.7 \\
\hline
\end{tabular}

Table 4: Comparison of lamotrigine pharmacokinetics obtained by Arunee Srichaiya et al. [23] and those obtained in this study. were reported. The adverse reactions reported by two volunteers were resolved spontaneously at the end of each period and were considered mild. In both cases the event was presented with the test product and was consistent with effects reported for lamotrigine.

Lamotrigine plasma concentrations over the quantification limit $(0.1 \mu \mathrm{g} / \mathrm{mL})$ were observed within 0.33 to 120 hours after drug administration. Although blood samples were taken until 144 hours plasma concentrations at this time showed levels of $0.0940 \pm 0.0329$ $\mu \mathrm{g} / \mathrm{mL}$ and $0.0864 \pm 0.0442 \mu \mathrm{g} / \mathrm{mL}$ for the test and reference product, respectively. These values were not used to calculate pharmacokinetic parameters.

Pharmacokinetics parameters obtained in this research were similar to those found by Srichaiya et al. [23] in a study with the same drug, at the same dose in a group of healthy Thai male volunteers, using an HPLC method with high sensitivity LOQ $(0.05 \mu \mathrm{g} / \mathrm{mL})$ as shown in Table 4

The values obtained for $90 \%$ CIs for the mean ratio (test/reference) of $\mathrm{AUC}_{0-\infty}$ and $\mathrm{C}_{\max }$ (Table 3) fell within the $80 \%-125 \%$ interval establish as bioequivalence limit [24]. It was concluded that the tested LAMOTRIGINE tablet (100 mg) elaborated by Humax Pharmaceutical S.A. is bioequivalent to the reference formulation, LAMICTAL $^{\circledR} 100$ mg, manufactured by Glaxo operations UK Ltd. in terms of rate and amount of absorption, according to the guidances of Instituto Nacional de Vigilancia de Medicamentos y Alimentos (INVIMA) and FDA.

\section{Acknowledgement}

This study was sponsored by Humax Pharmaceutical, contract no. CPS 121 de 2007. We thank Professor Abel Díaz by performing statistical analysis, Lucia Fernanda Jaime Claro, Pharmaceutical Chemistry student, for her cooperation in the development of clinical stage and David Galvis Pareja, Ph.D for his suggestions and advice in writing this paper. We disclose no conflict of interest with Glaxo Operations UK Ltd.

\section{References}

1. Bhagwagar Z, Goodwin GM (2005) Lamotrigine in the treatment of bipolar disorder. Expert Opin Pharmacother 6: 1401-1408.

2. Bowden $\mathrm{CL}$ (2002) Lamotrigine in the treatment of bipolar disorder. Expert Opin Pharmacother 3: 1513-1519.

3. Malik S, Arif H, Hirsch LJ (2006) Lamotrigine and its applications in the treatment of epilepsy and other neurological and psychiatric disorders. Expert Rev Neurother 6: 1609-1627.

4. Biton V, Sackellares JC, Vuong A, Hammer AE, Barrett PS, et al. (2005) Double-blind, placebo-controlled study of lamotrigine in primary generalized tonic-clonic seizures. Neurology 65: 1737-1743.

5. Yang YC, Hsieh JY, Kuo CC (2009) The external pore loop interacts with S6 and S3-S4 linker in domain 4 to assume an essential role in gating control and anticonvulsant action in the $\mathrm{Na}(+)$ channel. J Gen Physiol 134: 95-113.

6. Sitges M, Chiu LM, Guarneros A, Nekrassov V (2007) Effects of carbamazepine phenytoin, lamotrigine, oxcarbazepine, topiramate and vinpocetine on $\mathrm{Na}$ channel-mediated release of $[3 \mathrm{H}]$ glutamate in hippocampal nerve endings. Neuropharmacology 52: 598-605.

7. Garnett WR (1997) Lamotrigine: pharmacokinetics. J Child Neurol 12: 10-15

8. Peck AW (1991) Clinical pharmacology of lamotrigine. Epilepsia 32: S9-S12.

9. Cohen AF, Land GS, Breimer DD, Yuen WC, Winton C, et al. (1987) Lamotrigine, a new anticonvulsant: pharmacokinetics in normal humans. Clin Pharmacol Ther 42: 535-541.

10. Borlak J, Gasparic A, Locher M, Shupke H, Hermann R (2006) N-Glucuronidation of the antiepileptic drug retigabine: results from studies with human volunteers, 
Citation: Ruiz A, Cuesta F, Parra S, Montoya B, Restrepo M, Archbold R, et al. (2012) Bioequivalence Evaluation of Two Formulations of Lamotrigine Tablets in Healthy Volunteers. J Bioequiv Availab 4: 030-034. doi:10.4172/jbb.1000107

heterologously expressed human UGTs, human liver, kidney, and liver microsomal membranes of Crigler-Najjar type II. Metabolism 55: 711-721.

11. Rambeck B, Wolf P (1993) Lamotrigine clinical pharmacokinetics. Clin Pharmacokinet 25: 433-443.

12. Goa KL, Ross SR, Chrisp P (1993) Lamotrigine: a review of its pharmacological properties and clinical efficacy in epilepsy. Drugs 46: 152-176.

13. Metropolitan Life (1983) Desirable weights of adults. http://www.halls.md/idealweight/met.htm.

14. World Medical Association (2008) Declaration of Helsinki: Ethical principles for medical research involving human subjects.

15. European Medicines Agency (2005) International conference on harmonisation. Guideline for good clinical practice. ICH topic E6.

16. ORGANIZACIÓN PANAMERICANA de la SALUD - ORGANIZACIÓN MUNDIAL de la SALUD OPS/OMS (2002) Normas éticas internacionales para las investigaciones biomédicas con sujetos humanos.

17. Ministerio de Salud (1993). Normas científicas, técnicas y administrativas para la investigación en salud. Resolución No. 008430. Ministerio de Salud, BogotáColombia.
18. Matar KM, Nicholls PJ, Tekle A, Bawazir SA, Al-Hassan MI (1999) Liquid chromatographic determination of six antiepileptic drugs and two metabolites in microsamples of human plasma. Ther Drug Monit 21: 559-66.

19. Food and Drug Administration (2001) Guidance for industry, Bioanalytical method validation.

20. Schuirmann DJ (1987) A comparison of the two one-sided tests procedure and the power approach for assessing the equivalence of average bioavailability. $J$ Pharmacokinet Biopharm 15: 657-680.

21. Food and Drug Administration (2003) Guidance for industry, bioavailability and bioequivalence studies for orally administered drug products.

22. Julious SA (2004) Sample sizes for clinical trials with normal data. Stat Med 23: 1921-1986.

23. Srichaiya A, Longchoopol $\mathrm{CH}$, Oo-Puthinan $\mathrm{S}$, Sayasathid J, Sripalakit $P$, et al. (2008) Bioequivalence of generic lamotrigine 100-mg tablets in healthy Thai male volunteers: a randomized, single-dose, two-period, two-sequence crossover study. Clin Ther 30: 1844-1851.

24. Food and Drug Administration (2001) Guidance for industry, statistical approaches to establishing bioequivalence. 